\title{
UTILIZAÇÃO DAS FERRAMENTAS DA QUALIDADE ASSOCIADAS A APLICAÇÃO DAS METODOLOGIAS DE ENSINO.
}

Laysia Nayara da Silva Bezerra; laysianayara01@gmail.com; Instituto Federal de Educação

Ciência e tecnologia do Rio Grande do Norte;

Isamara de Melo Dantas Bezerra; isamaradantass@gmail.com; Instituto Federal de Educação Ciência e tecnologia do Rio Grande do Norte;

\section{Resumo}

No presente artigo é exposto algumas das ferramentas da qualidade e como elas podem contribuir para a melhora contínua do rendimento acadêmico de alunos que apresentam déficits. A partir da aplicação dessas ferramentas foi possível analisar a atual metodologia utilizada pelas redes de ensino e destacar alguns dos seus pontos relevantes para que a mesma não desempenhe com eficiência sua função. Para obter esses resultados foram utilizadas as ferramentas: Diagrama de Pareto; Diagrama de Ishikawa; Carta de Controle; Folha de Verificação; Diagrama de Dispersão; Histograma e Matriz GUT. A fim de exemplificar houve a implementação das ferramentas associadas a temática. A princípio foi utilizado o diagrama de Pareto, logo após a análise do gráfico pode-se identificar as meterias que necessitavam maior atenção e aplicou-se o diagrama de Ishikawa para que houvesse a identificação dos possíveis motivos para que tais matérias fossem tidas como de pior rendimento. Para que houvesse maior domínio do conteúdo em questão, se deu uma pesquisa do método de ensino VAK, que consiste no relacionamento de três canais receptores: visual, auditivo e cinestésico.

Palavras-Chaves: Ferramentas da qualidade; Métodos acadêmicos; Metodologias.

\section{Introdução}

As metodologias utilizadas no ensino independentemente da sua respectiva área abordada ou modalidade de ensino, dependem tanto de seu docente como do aluno para o desenvolvimento do aprendizado. Durante todo o período vivenciado na escola é normal que o estudante apresente dificuldades em determinadas matérias, visto que as ferramentas de ensino utilizadas pelos professores são análogas.

Para reparar as dificuldades relacionadas as deficiências de aprendizado já existem algumas metodologias e técnicas como, estudar o assunto antes das aulas, ou escrever o que se ouve durante as aulas. Mas ainda há dificuldades que essas metodologias não conseguem abranger. 
As ferramentas da qualidade consistem em metodologias e técnicas que são utilizadas para a resolução de obstáculos encontrados independente da sua natureza, visando buscar a melhora continua. Considerando essa perspectiva, a aplicação das ferramentas da qualidade utilizadas para auxiliar e resolver os obstáculos encontrados na aprendizagem dos estudantes seria um dos possíveis caminhos viáveis para atingir os objetivos pessoais dos alunos.

Neste presente artigo é proposto a utilização das ferramentas da qualidade associadas as metodologias de ensino, buscando solucionar as dificuldades encontradas pelos discentes e obter a melhoria contínua nos resultados obtidos por eles. Onde eles mesmos poderiam classificar as suas dificuldades e atribuir a elas seus respectivos níveis de priorização.

Nesse sentido, o presente trabalho busca descrever como as ferramentas da qualidade podem contribuir para a identificação de metodologias no âmbito acadêmico, incentivando os alunos a buscarem os motivos de suas dificuldades, e novos meios de como resolvê-las, de forma que haja a auto avaliação acerca de seus atuais comportamentos, visando um melhor desenvolvimento de seu desempenho acadêmico.

\section{Desenvolvimento}

Atualmente se é comum a aplicação de novas metodologias no ensino acadêmico, visando a melhor forma de aprendizagem para os alunos, uma vez que os mesmos dependem das ferramentas e técnicas pedagógicas utilizadas no âmbito acadêmico pelos professores. Tais metodologias como: projeção de slides; estudos de filmes; livros didáticos; aulas práticas; e aulas de campo.

Porém em alguns casos a utilização das metodologias citadas acima não são suficientes para sanar as dúvidas e deficiências dos estudantes em questão, é importante que se leve em consideração as especificidades de cada aluno em relação a sua vida acadêmica, para que haja uma melhor análise e desenvolvimento das barreiras identificadas ao decorrer de seus estudos. É imprescindível que se atualize as atuais ferramentas utilizadas no ensino, visando que os discentes alcancem um desempenho de maior eficácia durante o período acadêmico exercido por ele. Buscando reduzir ou até mesmo cessar os bloqueios identificados.

É importante que o aluno possua capacidade de criar formas para melhorar seu desempenho e que possa utilizar artifícios para alterar sua condição acadêmica. Para isso é indispensável que o discente esteja disposto a abdicar de momentos como: Dedicar maior 
parte de seu tempo a sua vida social. Dado que a ausência de organização é um ponto crucial para a um desempenho indesejado em quaisquer áreas, em especial no meio acadêmico.

\section{Fundamentação}

\subsection{Ferramentas da qualidade}

As ferramentas da qualidade são metodologias e técnicas utilizadas na gestão da qualidade, onde são aplicadas em ocasiões para a resolução de obstáculos encontrados. Ao serem utilizadas de maneira correta, as mesmas atribuirão para a organização uma série de benefícios, sendo eles: uma diminuição de despesas, melhor execução de atividades operacionais e cooperação dos profissionais envolvidos.

Além da identificação de problemas em projetos, fazendo com que haja uma elevação da qualidade no serviço logístico. "São ferramentas simples e muito eficazes que auxiliam o gestor na solução de problemas e ainda permitem o crescimento do ser humano em direção da qualidade de vida, pois também pode utilizadas a esfera pessoal.” (SELEME, Robson; STADLER, Humberto. 2008)

\subsection{Diagrama de Pareto}

O diagrama de Pareto foi desenvolvido por Vilfredo Pareto. O desenvolvimento dessa técnica partiu do princípio de que $80 \%$ da riqueza de algumas populações correspondem a $20 \%$ das pessoas e que $20 \%$ da riqueza corresponde a $80 \%$ da população. "O diagrama de Pareto é uma forma de descrição gráfica onde se procura identificar quais os itens responsáveis pela maior parcela dos problemas" (BEHR et al., 2008). A curva ABC, 8020,70-30 ou diagrama de Pareto é uma ferramenta utilizada para a priorização de ocorrências, onde pode-se visualizar em seu gráfico os problemas, seu número de ocorrências e suas perspectivas porcentagens. 
Figura 2 - Diagrama de Pareto.

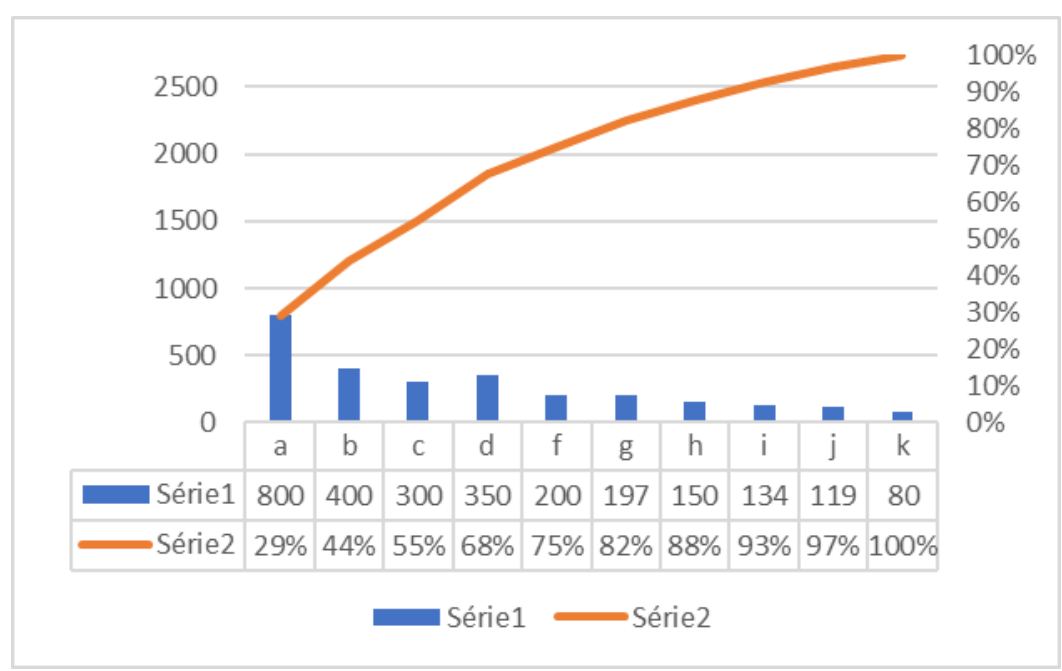

Fonte: Autores (2020)

\subsection{Diagrama de Ishikawa}

O diagrama de causa e efeito, também conhecido como "espinha de peixe" por sua forma de visualização é uma ferramenta que busca analisar as possíveis causas do problema encontrado. Em 1943 Kaoru Ishikawa criou o diagrama que acabou recebendo o seu nome. O diagrama consiste em uma técnica virtual que possibilita que haja uma interligação dos resultados (efeitos com os fatores (causas). O mesmo é um instrumento utilizado para estudar os fatores que determinam resultados e as causas dos problemas que precisamos evitar ou solucionar.

Figura 3 - Diagrama de Ishikawa.

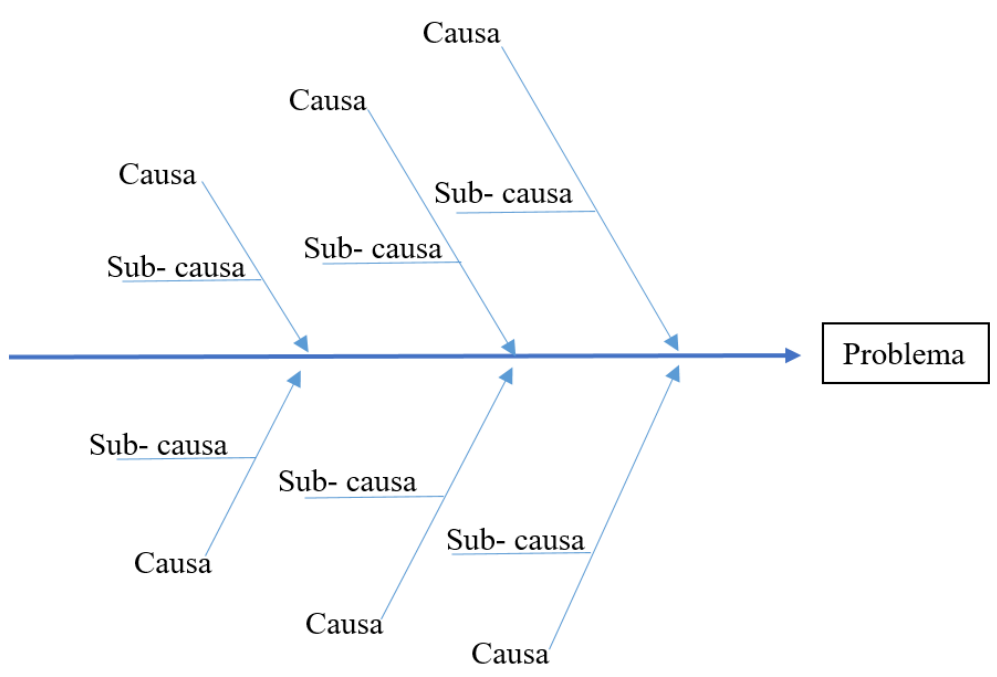

Fonte: Autores (2020). 
Segundo Slack et al (2002) define-se que devem existem quatro etapas de montagem do diagrama de Ishikawa: a primeira deve ser elencar o problema na caixa de efeito/problema; na segunda acontece a identificação das principais razões para as possíveis causas do problema; a terceira deverá ocorrer a busca de fatores em discussões de grupos que irão gerar possíveis causas para o problema realmente; e por último a quarta fase ou parte do processo, é o registro de todas as causas potenciais no diagrama, discutindo cada item e procurando esclarecimento das causas.

\subsection{Carta de controle}

Walter Shewhart, na década de 20, foi o primeiro a formular a distinção entre variação controlada e não controlada, que se referente ao que se denomina de causas comuns e causas especiais. De acordo Pithon (2014) Shewhart desenvolveu uma ferramenta simples, utilizada para separação desses dois tipos de causas, que chamou de carta de controle.

Figura 4 - Carta de controle.

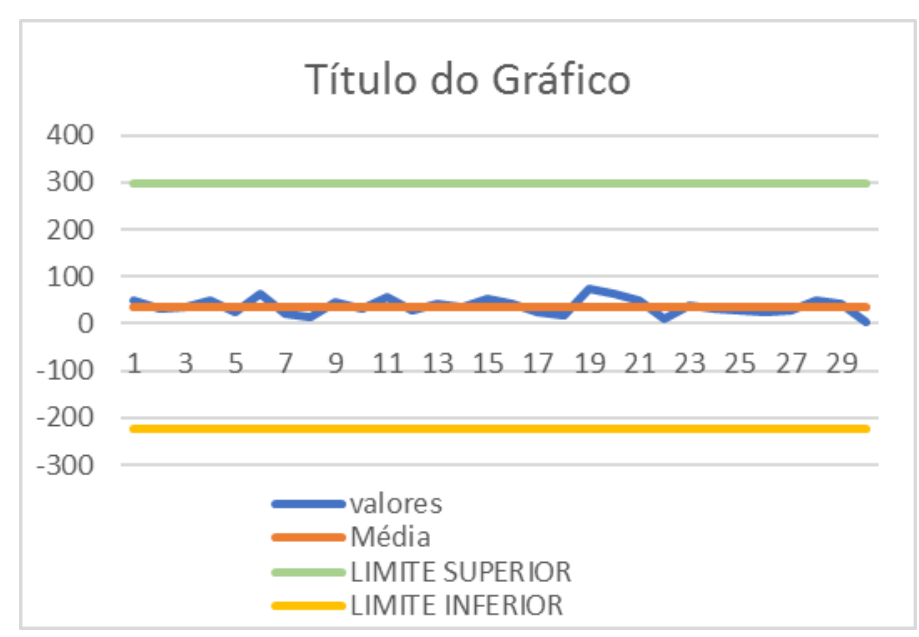

Fonte: Autores (2020).

A carta de controle assume um papel fundamental no processo de gerenciamento, permitindo que a empresa monitore e controle os dados a partir do gráfico gerado. Este gráfico determina uma faixa denominada limites de controle que são limitadas através da linha superior (limite superior de controle) e inferior (limite inferior de controle), além de uma linha média.

\subsection{Folha de verificação}

Consiste em tabelas ou planilhas utilizadas para facilitar a obtenção de dados em um formato sistemático para compilação e análise. Sua utilização possibilita a otimização de 
tempo, pois elimina o trabalho de se desenharem figuras ou escrever números repetitivos, evitando comprometer a análise dos dados.

Segundo Valle (2007) serve para a observar fenômenos, possibilitando uma visualização da existência dos diversos fatores envolvidos e seus padrões de comportamento. De acordo com Valle (2007) há diversos tipos de listas para a verificação, variando da sua adaptação para as finalidades a que se destinam.

Tabela 1 - Folha de verificação

\begin{tabular}{|c|c|c|c|c|c|c|}
\hline Peças & Segunda & Terça & Quarta & Quinta & Sexta & $\begin{array}{c}\text { Total de } \\
\text { falhas }\end{array}$ \\
\hline Objeto 1 & 2 & 5 & 3 & 0 & 8 & 18 \\
\hline Objeto 2 & 4 & 2 & 7 & 4 & 3 & 20 \\
\hline Objeto 3 & 6 & 3 & 8 & 7 & 2 & 26 \\
\hline Objeto 4 & 0 & 2 & 2 & 9 & 5 & 18 \\
\hline Objeto 5 & 4 & 3 & 7 & 0 & 2 & 16 \\
\hline
\end{tabular}

Fonte: Autores (2020).

A aplicação da folha de verificação está relacionada com a observação de ocorrências. O número de acontecimentos de um problema ou de um evento é registrado na folha, de forma simplificada. Essa ferramenta também é utilizada em situações em que participantes de uma reunião, após o trabalho de identificação e do agrupamento das causas de um problema ou de alternativas para a sua solução, indiquem aquelas que pareçam ser as mais significativas.

\subsection{Diagrama de dispersão}

O diagrama de dispersão é utilizado para a verificação de correlação entre duas variáveis. Segundo Costa (1972) quando uma variável tem o seu valor rebaixado com o aumento da outra, diz-se que as mesmas são negativamente correlacionadas. Pode-se também determinar a reta no gráfico que melhor se ajusta aos pontos do diagrama de dispersão. Essa reta é denominada de linha de tendência. De acordo Meireles (2001) a reta serve para visualizar a correlação do médio linear entre as duas variáveis. 
O diagrama pode ser usado em ocasiões em que haja a necessidade de se identificar possíveis raízes de problemas, de dois efeitos que ocorrem a partir do mesmo efeito ou ao testar a autor relação antes de construir um gráfico de controle.

Figura 5 - Diagrama de dispersão

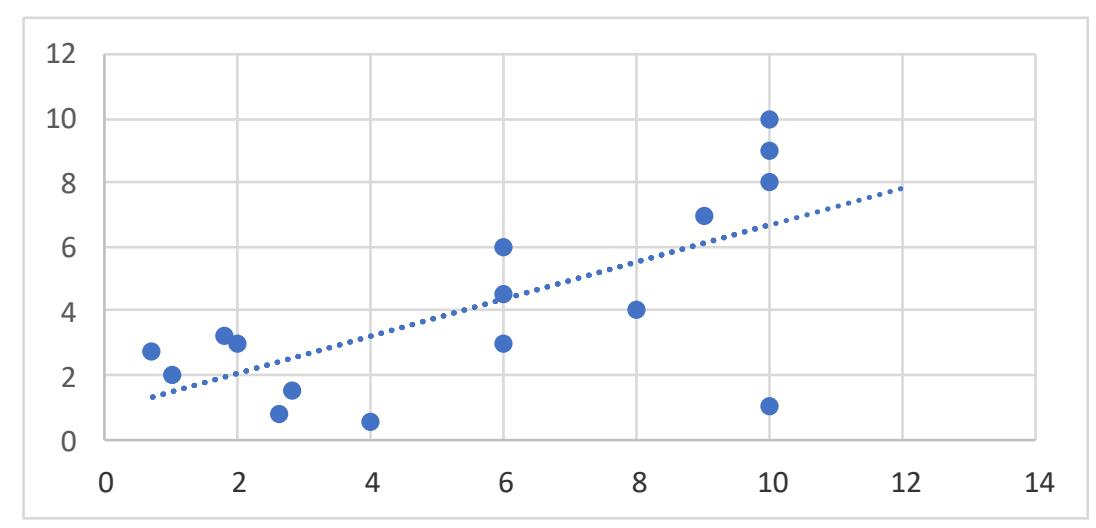

Fonte: Autores (2020).

\subsection{Histograma}

Consiste em uma ferramenta estatística gráfica agrupada em classes de frequência que permitem a verificação da forma de distribuição, o valor central e a dispersão dos dados. Segundo Braz (2002) o histograma é um gráfico composto por retângulos contínuos com bases nas faixas de valores das variáveis em estudo e cuja altura é dada por meio da frequência das ocorrências dos dados no intervalo definido pela base do retângulo.

Figura 6 - Histograma

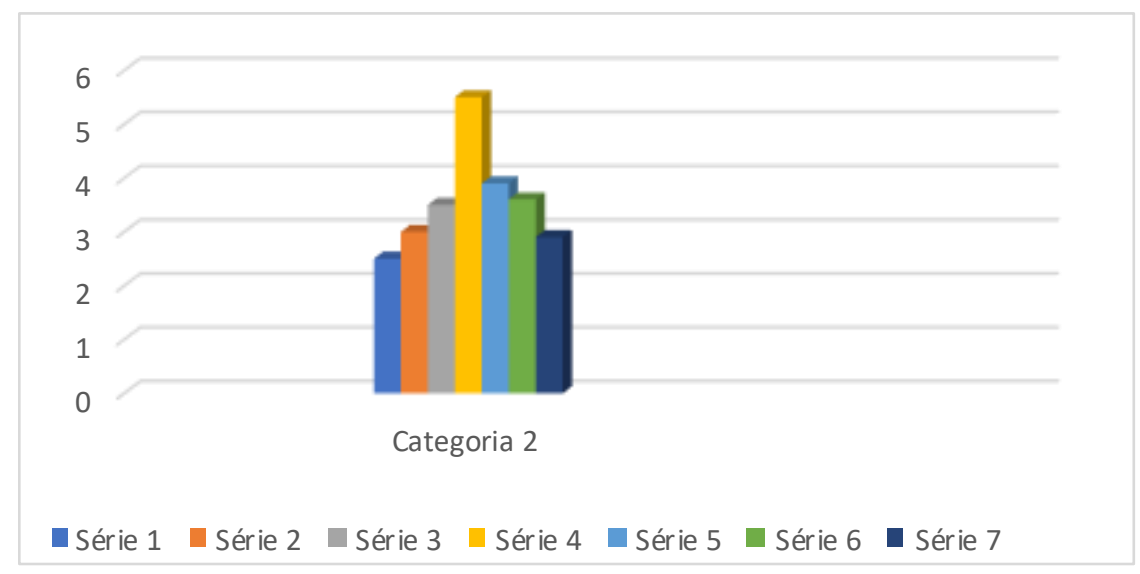

Fonte: Autores (2020).

O histograma é um gráfico de barras verticais que apresenta valores de uma certa característica agrupados por faixas. É útil para identificar o comportamento típico das 
características de tendências, permitindo a visualização de determinados acontecimentos, dando uma noção da frequência com que ocorrem.

\subsection{Matriz de priorização de gravidade, urgência e tendência GUT}

A ferramenta GUT consiste na priorização de problemas encontrados, possibilitando uma melhor tomada de decisão, considerando a gravidade, urgência e tendência dos problemas encontrados onde lhes são atribuídas notas de 1 a 5, sendo 5 a nota máxima, possibilitando visualização clara de prioridade entre os problemas. Segundo Da Hora, Henrique Rego Monteiro (2009) A técnica da Matriz de priorização possui como principal vantagem sua facilidade de uso e agilidade na obtenção de resultados.

Tabela 2 - Matriz GUT

\begin{tabular}{|c|c|c|c|c|}
\hline Problema & Gravidade & Urgência & Tendência & Nota \\
\hline$X$ & 4 & 4 & 2 & 32 \\
\hline $\mathrm{Y}$ & 5 & 4 & 4 & 80 \\
\hline
\end{tabular}

Fonte: Autores (2020)

A matriz GUT possibilita fazer uma análise do ambiente interno e externo da empresa. A matriz GUT é uma ferramenta utilizada para pelas empresas na gestão de projetos e tomada de decisões estratégicas, permitindo quantificar a problemas da organização ou tarefa a serem executadas.

\section{Metodologias de aprendizagem}

\subsection{Visual, auditivo, cinestésico (VAK)}

A metodologia VAK consiste no relacionamento dos três principais canais receptores sensoriais: visual, auditivo e cinestésico. Essa abordagem é utilizada de acordo com as preferencias individuais por um desses três meios de aprendizagem. Segundo Gallert, Cleia Scholles; Martins-Pacheco, Lúcia Helena (2005). O estilo de aprendizagem VAK é derivado do universo da aprendizagem ativa e baseia-se no uso dos três canais sensoriais: visão, audição e cinestésico (tato e movimento). As pessoas ditas normais percebem o mundo por meio destes três canais sensoriais, porém existe preferência por um dos canais durante o processo de aprendizagem. 
A metodologia diferenciada é muito relevante, dado que há pessoas que respondem a determinado sentido. O método VAK (VISUAL, AUDITIVO e CINESTÉSICO) é tem embasamento nos sentidos. Essa teoria, foi construída partindo da perspectiva que o aprendizado dos alunos se dá a partir de estímulos visuais, auditivos e cinestésicos, ou seja, cada estudante possui uma predominância em determinado estilo ou até mesmo uma mistura moderada dos três estilos: visual, auditivo e cinestésico. A mesma foi desenvolvida por Fernald, Keller, Orton, Gilingham, Stillman e Montessori. Cada estimulo tem suas características especificas, tais como:

- Estilo Visual: Neste grupo encontra-se estudantes que possuem facilidade em diferenciar e interpretar estímulos visuais. Através da visualização de imagens e figuras, é possível determinar ligações entre ideias e concepções

- Estilo Auditivo: Os estudantes que apresentam o estilo auditivo dispõem de habilidades como reconhecer a estímulos como sons e ruídos. Formam suas ideias a partir da interação direta com estímulos auditivos.

- Estilo Cinestésico: Nesse grupo é encontrado estudantes que utilizam dos estímulos recebidos pelos movimentos corporais.

\section{Metodologia}

O presente artigo tem como metodologia, a pesquisa bibliográfica possuindo caráter qualitativo. $\mathrm{O}$ estudo foi feito a partir de artigos científicos, revistas e livros da área para o melhor esclarecimento. De acordo Gil (2002) a pesquisa bibliográfica se é desenvolvida no baseamento de materiais já elaborados, constituídos majoritariamente de livros e artigos científicos. O levantamento dos dados foi baseado em pesquisas no Google Acadêmico e Periódicos Capes. Os softwares utilizados foram: Word e Excel.

\section{Aplicação das ferramentas}

Considerando a perspectiva de que o aluno ao encontrar obstáculos em sua aprendizagem em determinadas áreas, o mesmo deve buscar medidas para ir antemão a suas dificuldades. Partindo da necessidade de conseguir reparar o déficit dos estudantes, o presente artigo propõe a implementação das ferramentas da qualidade associadas ao âmbito escolar, possibilitando um melhor controle de suas ações direcionadas ao ambiente acadêmico. Para exemplificar de forma expositiva suponhamos a situação em que o estudante se encontre em dificuldades nas matérias de: matemática, física, química e biologia. Inicialmente deve ser realizada a análise das notas ao longo bimestre, possibilitando a classificação das matérias de 
acordo com a sua porcentagem apresentada no gráfico de Pareto, para que seja feita a priorização de maneira que se adeque as necessidades do aluno.

Figura 7 - Aplicação do gráfico de Pareto

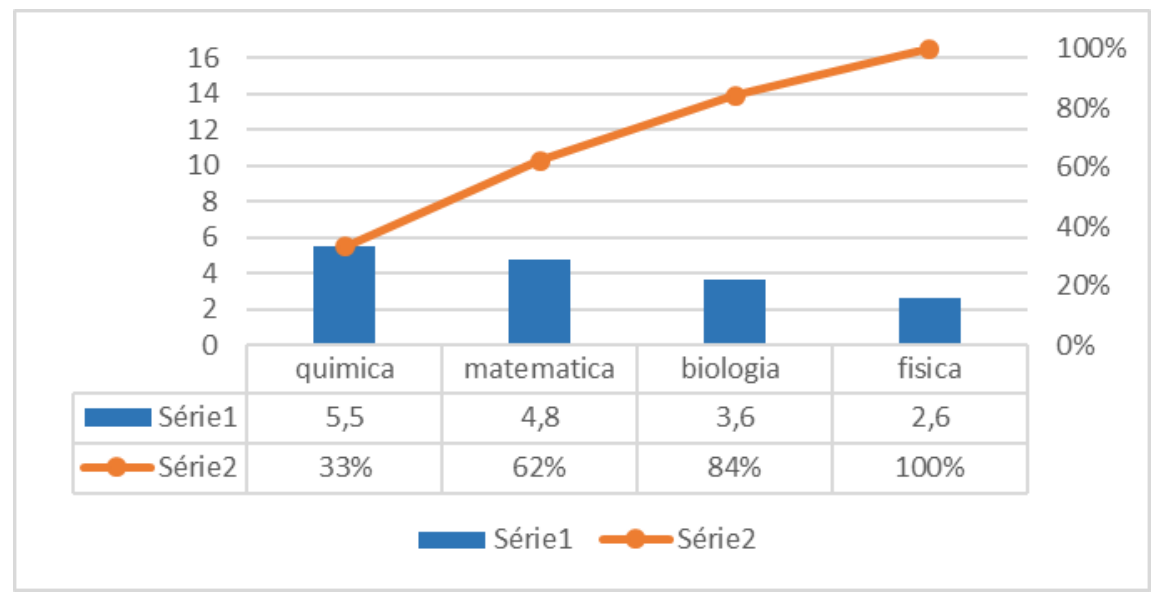

Fonte: Autores (2020)

Após a análise do gráfico de Pareto apresentado na figura 7 pode-se identificar as matérias que necessitam maior atenção, pois possuem menor índice de desenvoltura por parte do aluno. Em seguida o diagrama de Ishikawa seria ideal para elencar as principais causas.

Figura 8 - Aplicação de diagrama de Ishikawa

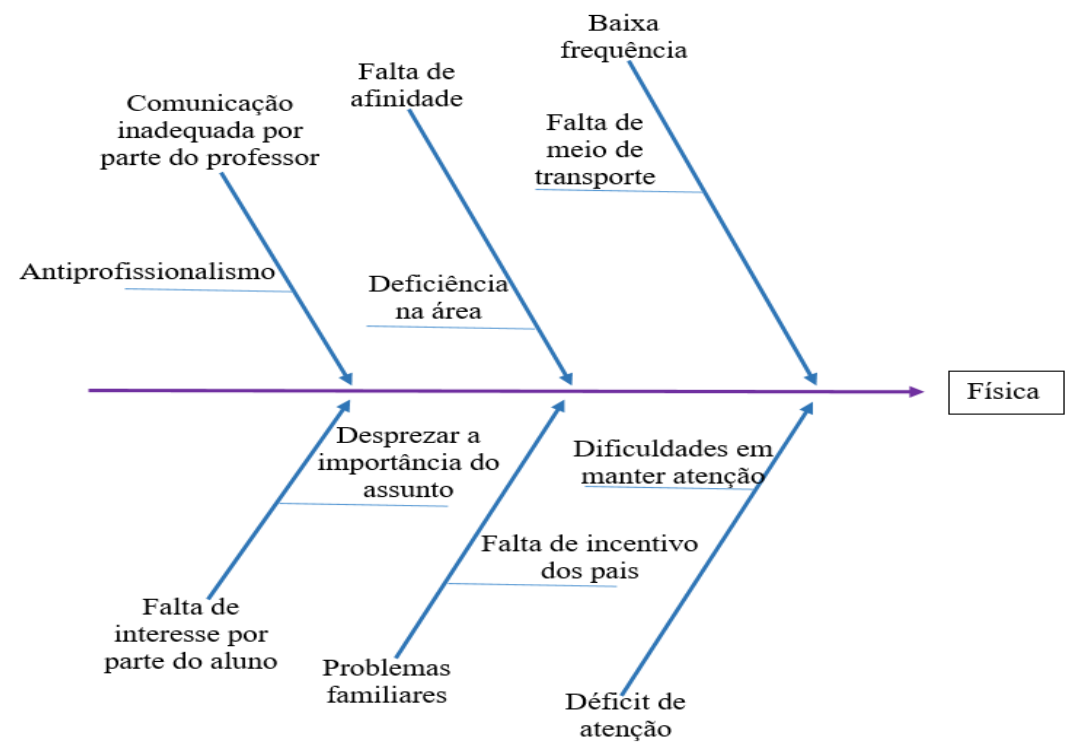

Fonte: Autores (2020).

Com a aplicação do diagrama de Ishikawa apresentado acima, podemos constatar as principais razões para que a matéria de física se torne um obstáculo para o discente. Facilitando a resolução das principais causas identificadas, logo solucionando o problema ao todo. 


\section{Análise}

A atual metodologia de ensino empregada em âmbito acadêmico não favorece de maneira integral a garantia de aprendizado do aluno, uma vez que não é oferecido às mesmas medidas de apoio que incentivem e busquem uma melhor desenvoltura em seu desempenho escolar.

Partindo da necessidade de oferecer ao estudante autonomia necessária para que o mesmo seja capaz de identificar falhas em seus métodos de aprendizagem, nesse artigo é proposto e exemplificado meios de identificar os empecilhos por meio da aplicação das ferramentas da qualidade, pois as ferramentas consistem em analisar e identificar falhas e erros, buscando a melhora continua.

Ao aplicarmos as ferramentas da qualidade, como foi visto no tópico 5, se obtém uma percepção mais ampla dos reais motivos que ocasionam uma disfunção no desempenho dos estudantes. A implementação das mesmas possibilita ao aluno que o mesmo possa construir técnicas que melhorem o cenário educacional em que está inserido, levando em consideração a perspectiva de que cada estudante possui singularidades que os distingue um dos outros.

\section{Considerações finais}

Nesse trabalho foi exposto as ferramentas da qualidade, vale ressaltar que não foram expostas todas, e que sua utilização em conjunto proporcionam a obtenção de melhores resultados. Foi visto a atual metodologia aplicada no ensino e suas deficiências para com o aluno.

A partir da aplicação das ferramentas foi destacado alguns pontos crucias que normalmente não são observados como relevantes, abrindo espaço para que haja pequenas rupturas na conjuntura da metodologia. As ferramentas associadas aos métodos de ensino têm o intuito de ir antemão aos bloqueios encontrados, corrigindo os mesmos e fortalecendo o aprendizado do discente.

Ao possibilitar aos estudantes a autossuficiência necessária para o desenvolvimento de técnicas que se adequem a suas respectivas necessidades, não só é ofertado aos alunos um meio de melhorar seu desempenho, mas também a instituição ou órgão de ensino tem a possibilidade de elevar no seu nível de educação, atingindo um novo diferencial em comparação as demais instituições. 
Levando-se em consideração que as abordagens de ensino utilizadas atualmente possuem falhas e que exercem um papel importante no desempenho escolar, sendo ele negativo ou positivo. Nessa perspectiva é fundamental que haja uma intervenção, para que os problemas apresentados sejam solucionados de modo rápido e eficaz. Visando que o aluno não venha a adquirir desproporções em seu aprendizado. Portanto a implementação das ferramentas da qualidade associadas ou ensino se mostram eficientes, pois as mesmas buscam a melhora continua, independente das áreas que sejam aplicadas.

\section{Referências}

BEHR, A. et al. Gestão da biblioteca escolar: metodologias, enfoques e aplicação de ferramentas de gestão e serviços de biblioteca. Revista Ciência da Informação, v.37, n.2, p.3242,2008

BRAZ, M.A. Ferramentas e Gráficos Básicos. In: RONTONDORO, R.G.(Org) Seis Sigma : Estratégia Gerencial para a Melhoria de Processos, Produtos e Serviços. São Paulo: Atlas, 2002

COSTA, Adelino Amaro. Criatividade na Escola. Relatório de Mesa redonda no âmbito do Programa de Trabalhos do CERI/CDE. Lisboa: Gabinete de estudos e Planejamento da ação educativa, 1972 .

DA HORA, Henrique Rego Monteiro; UFF, Helder Gomes Costa. Tomada de decisão no MASP: uma contribuição para decisões utilizando a matriz AHP. 2009.

GALLERT, Cleia Scholles; MARTINS-PACHECO, Lúcia Helena. Sistema Hipermídia Baseado no Estilo de Aprendizagem VAK. VII Simpósio Internacional de Informática Educativa-SIIE05. Leiria, Portugal, p. 16-18. 
K Ishikawa - Recuperado el, 1943 - gerenciaprocesosucb.pbworks.com

MEIRELES, Manuel. Ferramentas administrativas para identificar, observar e analisar problemas. São Paulo: Arte \& Ciência, 2001.

PITHON, Roberta. Revista Banas. Retirado de:

http://www.banasqualidade.com.br/2012/portal/index.asp. Acesso em: 15/08/2019.

SELEME, Robson; STADLER, Humberto. Controle da qualidade: as ferramentas essenciais. Editora Ibpex, 2008.

SLACK, N.; CHAMBERS, S.; JOHNSTON, R. Administração da Produção. São Paulo: Atlas, 2002.

VALLE, José Angelo. 40 Ferramentas e Técnicas de Gerenciamento. Rio de Janeiro: Brasport, 2007 